\title{
Co-stimulation with stem cell factor and erythropoietin enhances migration of $c$-Kit expressing cervical cancer cells through the sustained activation of ERK1/2
}

\author{
CRISTINA AGUILAR ${ }^{1}$, CECILIA AGUILAR ${ }^{1}$, REBECA LOPEZ-MARURE ${ }^{2}$, \\ ALEJANDRO JIMÉNEZ-SÁNCHEZ ${ }^{1}$ and LETICIA ROCHA-ZAVALETA ${ }^{1}$
}

${ }^{1}$ Instituto de Investigaciones Biomédicas, Departamento de Biología Molecular y Biotecnología, UNAM, Ciudad Universitaria,
C.P. 04510; ${ }^{2}$ Instituto Nacional de Cardiología ‘Ignacio Chávez’, Departamento de Biología Celular,
Colonia Sección 16, Tlalpan, C.P. 14080, Mexico City, Mexico

Received July 30, 2013; Accepted February 17, 2014

DOI: $10.3892 / \mathrm{mmr} .2014 .2044$

\begin{abstract}
The cytokines erythropoietin (Epo) and stem cell factor (SCF), coupled with the cooperation between their receptors (EpoR and c-Kit), are essential components of normal physiological erythropoiesis. In earlier studies, we demonstrated the expression of c-Kit and EpoR in cervical cancer cells. It was identified that SCF is a survival factor, whereas Epo promotes cell proliferation. Cooperation between EpoR and SCF in cervical cancer has rarely been studied, despite the fact that cell migration and anchorage independent growth are considered initial steps in metastasis. Thus, the aim of this study was to analyse the effect of SCF and Epo alone, or in combination, on the migration and anchorage independent growth of two cervical cancer-derived cell lines. First, we demonstrated the expression of EpoR and c-Kit in the cell lines. Next, we evaluated anchorage independent growth, and identified that Epo and SCF produced a modest number of colonies, whereas the combination Epo/SCF induced a significantly higher number of colonies. Migration was then evaluated in Boyden chambers. Co-stimulation with Epo/SCF induced a significantly higher number of migrating cells than either cytokine alone. SCF-, Epo- and Epo/SCF-induced migration was inhibited by blocking phosphorylation of Janus kinase 2 (JAK2). Accordingly, western blot analysis demonstrated that the JAK2/signal transducer and activator of transcription-5 (STAT5) axis was activated in all cases. By contrast, inhibition of extracellular signal-related kinase (ERK) 1/2 abrogated migration induced by SCF and Epo/SCF only. Concurrently, Epo induced a modest, transient
\end{abstract}

Correspondence to: Dr Leticia Rocha-Zavaleta, Instituto de Investigaciones Biomédicas, Departamento de Biología Molecular y Biotecnología UNAM, Circuito Escolar S/N, Ciudad Universitaria, C.P. 04510, Mexico City, Mexico

E-mail:1rochaz@biomedicas.unam.mx

Key words: cervical cancer, erythropoietin, stem cell factor, c-Kit, cell migration activation of ERK1/2, whereas SCF and Epo/SCF prompted a strong, sustained phosphorylation of ERK1/2. The results from this study have revealed that co-stimulation with Epo/SCF promotes migration and anchorage independent cell growth, and that co-signalling from EpoR and c-Kit converge on JAK2/ STAT5 activation. Furthermore, SCF- and Epo/SCF-induced migration depends on the sustained activation of ERK1/2. These results indicate that co-signalling from different cytokine receptors induces migration, and this suggests that migratory behaviour may be regulated by the cooperative activity of Epo and SCF in cells expressing their cognate receptors.

\section{Introduction}

Erythropoietin (Epo) and stem cell factor (SCF) are key elements for normal erythropoiesis. Epo and SCF are important cytokines that regulate erythroid progenitor proliferation, survival and differentiation by binding to their receptors, EpoR and c-Kit, respectively. The interaction of Epo and EpoR induces the activation of EpoR-associated Janus kinase 2 (JAK2) by transphosphorylation. Activated JAK2 phosphorylates tyrosine residues on the cytoplasmic domain of the receptor (1). These phosphorylated residues may act as docking sites for a variety of Src homology-2 (SH2) domaincontaining proteins, initiating relevant signalling pathways, including the phosphatidylinositol-3 kinase (PI3K)/AKT, the mitogen-activated protein kinase (MAPK)/extracellular signal-related kinase (ERK) 1/2, and the JAK2/signal transducer and activator of transcription-5 (STAT5) pathways, promoting cell survival $(2,3)$ and proliferation (4).

On the other hand, binding of SCF induces the activation of c-Kit, a tyrosine kinase activity receptor, by transphosphorylation of various tyrosine residues. SH2 domain-containing signalling proteins are then recruited for the activation of transduction routes, including the PI3K/AKT cascade, which has been associated with the inhibition of apoptosis (5), the Src familiy kinase (SFK) signalling pathway, inducing cell proliferation (6), the MAPK/ERK route, implicated in the stimulation of cell migration (7), and the phospholipase C (PLC) and D (PLD) signalling cascade, which is involved in 
the protection against radiation-induced cell death (8) and the responses induced by membrane-bound SCF (9).

Cooperation between EpoR and c-Kit during erythropoiesis has been well documented (10-12). However, EpoR and c-Kit are also expressed in nonerythroid cells of the normal female genital tract $(13,14)$ and cervical tumours $(15)$. The effect of Epo and SCF on cervical cancer cells has been studied separately. In a previous study, we demonstrated the expression of functional c-Kit in cervical cancer cells, and presented evidence that SCF is a survival factor for this type of tumour cell (16). Following this, we described the presence of an autocrine/paracrine Epo/ EpoR system in cervical cancer cells, and demonstrated that exogenous Epo promotes cell proliferation in a JAK/STATdependent manner (17). In addition, it has been revealed that activation of EpoR may enhance the migration of cells derived from head and neck squamous cell carcinoma (18), breast cancer (19) and cervical cancer (20). Although cooperation between EpoR and c-Kit has been characterised in erythropoiesis (10-12), less is known about co-signalling between Epo and SCF in cancer. Cell migration is considered the first step in metastasis, therefore, identification of signalling proteins with the potential to contribute to cell migration may provide new insights into how cancer cell migration and metastasis are regulated. Thus, the aim of this study was to analyse the role of SCF, Epo and a combination of Epo/SCF on the anchorage independent cell growth and migration of cells derived from cervical tumours. We identified that co-stimulation of cervical cancer cells with Epo and SCF promotes migration and anchorage independent cell growth. This effect is significantly higher than that induced by either cytokine alone. Inhibition of JAK2 phosphorylation caused a significant reduction of Epo-, SCF- and Epo/SCF-induced migration. Similarly, western blot analysis demonstrated the activation of STAT5 in all treatments, suggesting that co-signalling from EpoR and c-Kit converge on JAK2/STAT5 activation. Furthermore, inhibition of ERK1/2 resulted in the abrogation of Epo-, SCF-, and Epo/SCF-induced migration. Western blot analysis demonstrated that stimulation with Epo induced a weak, transient activation of ERK1/2, whereas administration of SCF alone and Epo/SCF, induced a sustained activation of ERK1/2. Therefore, this suggests that co-stimulation with Epo/SCF may be regulating migration through the activation of multiple different signalling cascades.

\section{Materials and methods}

Cell lines and culture. Cervical cancer-derived cell lines were obtained as previously described (16). InBl cells were derived from a patient diagnosed with epidermoid, non-keratinized, metastatic cervical cancer (FIGO, stage IVB). The CaLo cell line was derived from a tumour biopsy from a patient diagnosed with epidermoid, non-keratinized cervical cancer (FIGO, stage IIB). The two cell lines were maintained in Dulbecco's modified Eagle's Medium (DMEM; Invitro, Mexico City, Mexico) supplemented with $2 \%$ foetal bovine serum (FBS; Invitrogen Life Technologies, Carlsbad, CA, USA). The K562 human chronic myelogenous leukaemia cell line was used as a positive control for the expression of EpoR (21). K562 cells were maintained in RPMI-1640 (Invitrogen Life Technologies) containing $10 \% \mathrm{FBS}$. All cells were incubated at $37^{\circ} \mathrm{C}$ in a humidified atmosphere of $95 \%$ air and $5 \% \mathrm{CO}_{2}$.
Western blot analysis. The cells were resuspended in lysis buffer (50 mM Tris-HCl, pH 7.4; 150 mM NaCl; 1 mM EDTA; $1 \%$ NP40; $0.25 \%$ sodium deoxycholate), containing $100 \mu \mathrm{l} / \mathrm{ml}$ complete protease inhibitors cocktail (Roche Applied Science, Mannheim, Germany) and $10 \mu \mathrm{l} / \mathrm{ml}$ phosphatase inhibitors (Sigma-Aldrich, St. Louis, MO, USA). For the isolation of membrane proteins, cells were resuspended in a lysis buffer containing $10 \mathrm{mM}$ Tris- $\mathrm{HCl}$ (pH 7.4), $50 \mathrm{mM} \mathrm{NaCl}, 5 \mathrm{mM}$ EDTA, $1 \%$ Triton X-100 and $0.05 \%$ SDS. Total protein content was determined using the DC protein assay kit (BioRad Laboratories, Hercules, CA, USA). The protein $(30 \mu \mathrm{g})$ was resolved by $10 \%$ SDS-PAGE and transferred onto polyvinylidene fluoride (PVDF) membranes (Millipore, Billerica, MA, USA). Membranes were incubated at $4^{\circ} \mathrm{C}$, overnight with specific antibodies diluted 1:1,000 and then washed and incubated with the appropriate horseradish peroxidase-conjugated secondary antibodies diluted 1:5,000 (Zymed Laboratories, Invitrogen Life Technologies, Carlsbad, CA, USA). For the detection of c-Kit, membranes were incubated with a biotinylated-swine antigoat, mouse and rabbit polyclonal antibody (DakoCytomation, Glostrup, Denmark) diluted 1:2,000 for $2 \mathrm{~h}$ at room temperature, followed by incubation with horseradish peroxidase-conjugated streptavidin (DakoCytomation) diluted 1:3,000 for $2 \mathrm{~h}$ at room temperature. Proteins were detected by chemiluminescence using the Amersham ECL plus Western Blotting Detection System (GE Healthcare Bio-Sciences, Piscataway, NJ, USA). For the detection of human EpoR, a goat anti-human EpoR antibody (Sigma-Aldrich) was used, which was produced using purified recombinant human erythropoietin soluble receptor as an immunogen. For the detection of c-Kit, a mouse anti-human c-Kit from Cell Signalling Technology Inc. (Danvers, MA, USA) was used. For the study of signalling cascades, rabbit anti-STAT5, rabbit anti-STAT5 (phospho Tyr 694), rabbit anti-ERK1 and ERK2 (ERK1/2) and rabbit anti-ERK (phospho Thr185 + Thr202 + Tyr204 + Tyr187) were used, all from GeneTex Inc. (Irvine, CA, USA). As an internal control, a rabbit anti-GAPDH (GeneTex Inc.) was included.

Flow cytometry. Cells were harvested, fixed in $2 \%$ paraformaldehyde, and stained with the carboxyfluorescein-conjugated mouse monoclonal anti-human erythropoietin receptor or phycoerythrin-conjugated mouse monoclonal anti-human c-Kit/CD117 (R\&D Systems, Minneapolis, MN, USA), diluted 1:4 in phosphate-buffered saline (PBS) for $1 \mathrm{~h}$. Cells were washed with PBS and analysed using a FACScalibur with Cell Quest software (Beckton Dickinson, San Jose, CA, USA).

Cell proliferation and survival assays. To evaluate cell proliferation, cells were incubated with different concentrations of human recombinant Epo (1, 10, 20, 30 and 50 U/ml; Sigma-Aldrich) or left untreated for 4 days. Proliferation was determined by the colorimetric MTT assay. To evaluate the effect of SCF on survival, the cells were incubated in the culture medium without FBS, treated with $15 \mathrm{U} / \mathrm{ml}$ of SCF, or left untreated for 14 days. Cell viability was evaluated by the MTT assay.

Cell migration assay. The effect of Epo and SCF on migration was evaluated using the $\mathrm{QCM}^{\mathrm{TM}}$ 24-well colorimetric cell migration assay (Chemicon International, Millipore, Temecula, CA, USA), which is based on the Boyden Chamber 
migration assay. The cells were starved by incubating $12 \mathrm{~h}$ prior to assay in FBS-free medium. Cells $\left(1 \times 10^{5}\right)$ were then seeded onto the upper chamber in $300 \mu \mathrm{l}$ of FBS-free medium and then supplemented with $5 \%$ bovine serum albumin (BSA). FBS-free medium $(500 \mu \mathrm{l})$ containing 5, 10, 20 and $30 \mathrm{U} / \mathrm{ml}$ Epo or SCF, or 10/20, 10/10 and 30/30 U/ml of Epo/ SCF combinations, was loaded into the lower chambers. As a negative control, FBS-free medium was used. Medium supplemented with $10 \%$ FBS was included as a positive control. The plates were incubated for $24 \mathrm{~h}$. Cell migration was determined by colorimetric measurement at $560 \mathrm{~nm}$.

Soft agar colony formation assay. Cells $\left(1 \times 10^{3}\right)$ were seeded in $0.3 \%$ agar in culture medium supplemented with 5\% FBS and $30 \mathrm{U} / \mathrm{ml}$ Epo or $15 \mathrm{U} / \mathrm{ml} \mathrm{SCF}$, or a combination of $30 \mathrm{U} / \mathrm{ml}$ Epo and $15 \mathrm{U} / \mathrm{ml} \mathrm{SCF}$, over a layer of $0.6 \%$ agar in 24-well plates. Following 7 days of incubation, colonies were stained with $1 \%$ crystal violet in $20 \%$ methanol, then imaged and counted.

Inhibition of receptors and signalling molecules. To inhibit the expression of EpoR in the cell membrane, the cells were incubated with $20 \mu \mathrm{M}$ lovastatin (Sigma-Aldrich), which is a selective inhibitor of the 3-hydroxy-3-methylglutaryl (HMG)-CoA reductase, for $20 \mathrm{~h}$ at $37^{\circ} \mathrm{C}$. Inhibition of surface expression was evaluated by western blotting of membrane protein extracts. For transient inhibition of c-Kit gene expression, a commercial small interfering RNA (siRNA) system was used (Santa Cruz Biotechnology Inc., CA, USA). Pools of target-specific 19-25 nucleotide siRNA (Santa Cruz catalogue no. 29225) were used to transfect cells according to the instructions of the manufacturer. As a negative control, cells were transfected with scrambled RNA. Cells were pre-incubated in antibiotic-free culture medium for $24 \mathrm{~h}$. siRNAs were diluted in transfection medium, mixed with the transfection reagent and incubated at room temperature for $30 \mathrm{~min}$. The mixture was overlaid onto the cells and then incubated for $7 \mathrm{~h}$ at $37^{\circ} \mathrm{C}$. Culture medium containing a double concentration of FBS and antibiotics was added without removing the transfection mixture and further incubated for $24 \mathrm{~h}$. The medium was replaced with fresh culture medium supplemented with $10 \%$ FBS and the cells were assayed $48 \mathrm{~h}$ later. Expression of c-Kit was evaluated by western blotting. To inhibit the JAK2 phosphorylation, the cells were incubated with $10 \mu \mathrm{M}$ Tyrphostin AG490 (Sigma-Aldrich) diluted in ethanol for $24 \mathrm{~h}$. PI3K was inhibited by incubating the cells with $100 \mathrm{nM}$ Wortmannin (Sigma-Aldrich) diluted in DMSO for $24 \mathrm{~h}$. To inhibit ERK1/2 kinases, the cells were incubated with $20 \mu \mathrm{M}$ U0126 ethanolate (Sigma-Aldrich) diluted in DMSO for $24 \mathrm{~h}$.

Isolation of c-Kit-expressing cells. Columns and reagents were purchased from Miltenyi Biotec (Teterow, Germany). For the isolation of c-Kit (also known as CD117) positive cells, the CD117 MicroBead kit, including paramagnetic microbeads conjugated to monoclonal mouse anti-human CD117 antibody, were used according to the instructions of the manufacturer. Briefly, $1 \times 10^{8}$ cells were resuspended in $300 \mu \mathrm{l}$ of column buffer, then $100 \mu \mathrm{l}$ of FcR blocking reagent and $100 \mu \mathrm{l}$ of CD117 MicroBeads were added, the cells were incubated for $15 \mathrm{~min}$ at $4^{\circ} \mathrm{C}$. Following washing of the cells, they were resuspended in $500 \mu \mathrm{l}$ of buffer. $\mathrm{LS}^{+}$columns were attached to the magnet and washed with $3 \mathrm{ml}$ of buffer, then the cell suspension was applied onto the column.
The column was washed three times and the unlabeled cells flowing through were collected for analysis. The column was removed from the separator magnet, $5 \mathrm{ml}$ of buffer was added onto the column, and magnetically labelled cells were flushed out by pushing the plunger into the column.

Statistical analysis. Results are presented as the mean \pm standard error (SEM). The t-test was used for the comparison between treatment groups and between cell lines. Confidence intervals (CI; 95\%) and P values were calculated. The test was two-tailed and $\mathrm{P}<0.05$ was considered to indicate a statistically significant result.

\section{Results}

Expression of EpoR and c-Kit in CaLo and InBl cells. Expression of EpoR and c-Kit was investigated in CaLo and InBl cells. Western blot analysis demonstrated the presence of a band of the predicted molecular weight for EpoR (65 kDa) and c-Kit (145 kDa) in CaLo and InBl cells (Fig. 1A). For EpoR to be functional, it must be translocated to the cell surface. Therefore, the expression of EpoR in the cell membrane was analysed by flow cytometry. As demonstrated in Fig. 1B, membrane expression of EpoR was detected in the CaLo and InBl cell lines. By contrast, detection of c-Kit by western blotting was problematic and required a biotin-mediated amplification step, suggesting that the receptor was either expressed at low levels or by a low number of cells. Therefore, the proportion of c-Kit expressing cells were evaluated by flow cytometry. As observed in Fig. 1B, a population of $12.8 \%$ of CaLo cells and $11.4 \%$ of InBl cells revealed a positive membrane expression of $\mathrm{c}-\mathrm{Kit}$.

Exogenous Epo stimulates proliferation of CaLo and InBl. In a previous study, we identified that exogenous Epo induces proliferation of cervical cancer cell lines. Therefore, to investigate whether stimulation with Epo induces proliferation of CaLo and $\mathrm{InBl}$, the cell lines were incubated in the presence of increasing concentrations of Epo. As expected, the cell lines demonstrated a dose-dependent increase in cell proliferation (Fig. 2A). However, proliferation of CaLo cells was significantly augmented from doses $>30 \mathrm{U} / \mathrm{ml}$, whereas enhancement of InBl proliferation was evident from doses $>1 \mathrm{U} / \mathrm{ml}$. In fact, throughout the various concentrations of Epo tested, proliferation of InBl was constitutively higher than that of CaLo (Fig. 2A). To verify that cell proliferation was mediated by EpoR, the cells were pre-incubated with lovastatin, which reduced the cell membrane expression of EpoR (Fig. 2B). As summarized in Fig. 2C, incubation with lovastatin reverted the proliferation effect induced by $30 \mathrm{U} / \mathrm{ml}$ of Epo, demonstrating that proliferation was mediated by EpoR.

Exogenous SCF induces survival of cells in the absence of $F B S$. Inhibition of endogenous SCF expression induces apoptosis in CaLo and InBl cells (16). In the present study, it was investigated whether exogenous SCF would protect cells from starvation-induced death. As observed in Fig. 2D, a small but significant proportion of CaLo cells survived FBS withdrawal when cultured in the presence of SCF. Of note, InBl cells were able not only to survive but also to proliferate as a response to SCF. Inhibition of c-Kit expression using siRNA (Fig. 2E) completely eliminated the effect of SCF (Fig. 2F). These obser- 
A
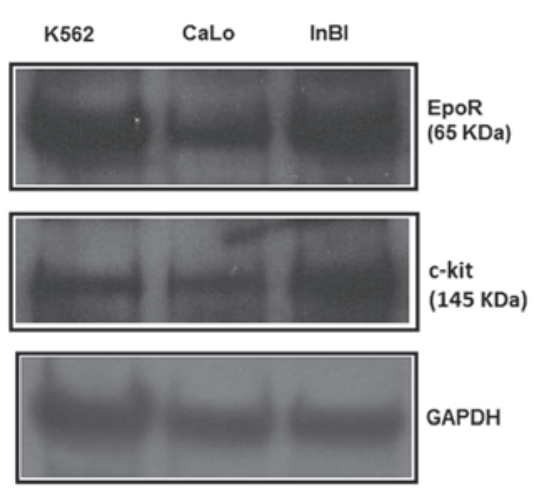

B
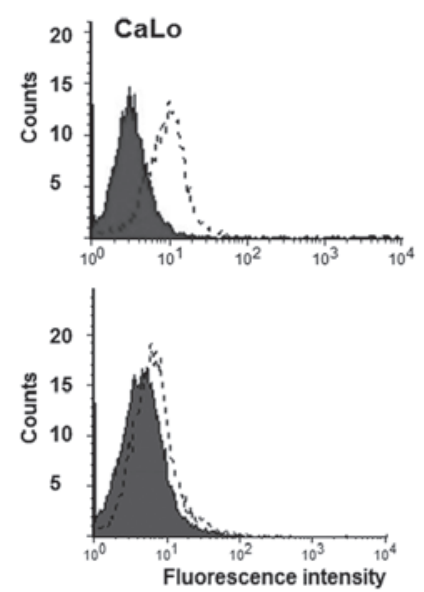
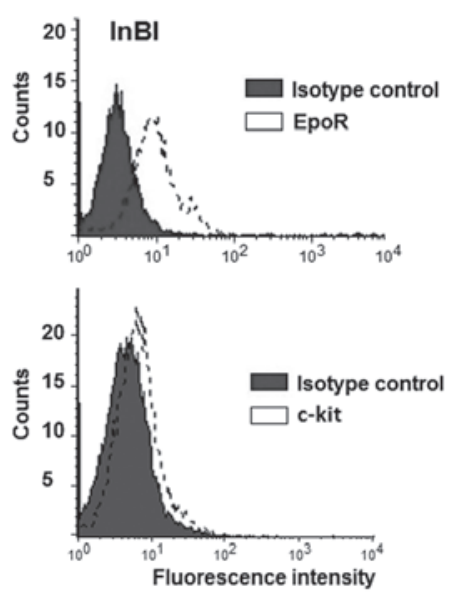

Figure 1. Expression of EpoR and c-Kit in cervical cancer cell lines. (A) Expression of EpoR and c-Kit was analysed in membrane protein extracts from CaLo and $\mathrm{InBl}$ cells by western blotting. EpoR was detected with an antibody produced using human erythropoietin soluble receptor as an immunogen. c-Kit could only be detected by using a biotin/streptavidin-mediated signal amplification step, following incubation with a monoclonal anti-human c-Kit antibody. Human leukaemia K562 cells were used as the positive control. (B) Cell surface expression of EpoR and c-Kit in CaLo and InBl cells was evaluated by flow cytometry. As a negative control, a primary isotype control antibody was used instead of either anti-EpoR or anti-c-Kit antibodies. EpoR, erythropoietin receptor.

A

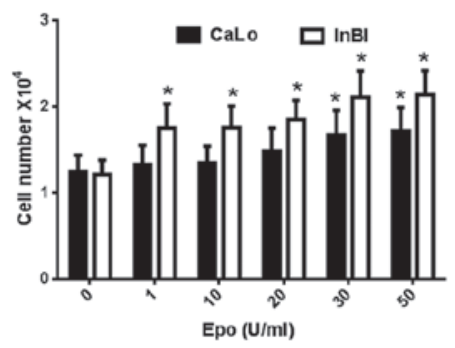

C

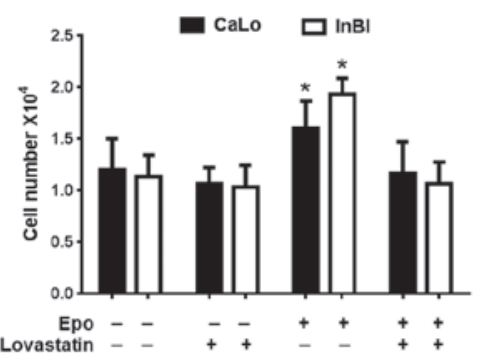

E

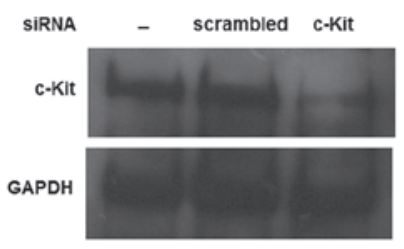

CaLo

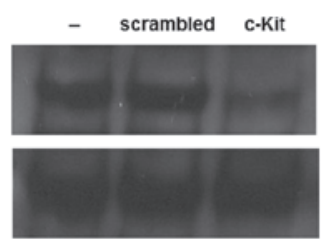

InBI
B

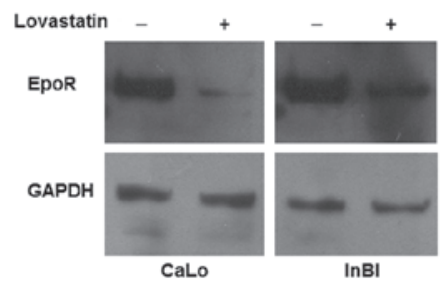

D
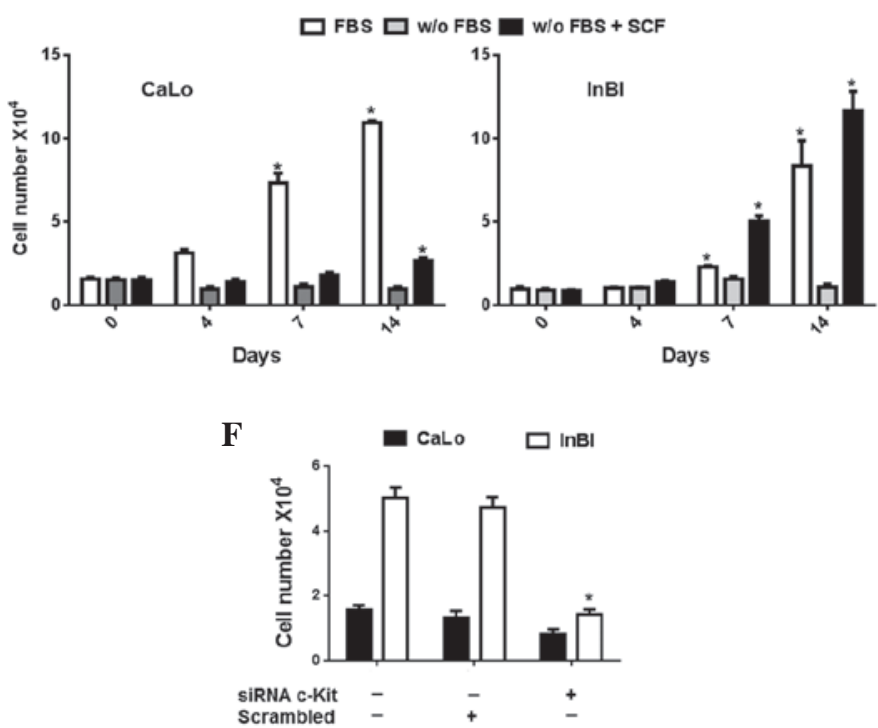

Figure 2. Evaluation of the effect of Epo and SCF on cervical cancer cells. (A) CaLo and InBl cells were incubated with the indicated concentrations of Epo. Cell number was evaluated by the colorimetric MTT assay. (B) To demonstrate that Epo-induced proliferation was mediated by cell membrane EpoR, the cells were pre-incubated with lovastatin. The effect of lovastatin on the cell surface expression of EpoR was investigated by western blotting of membrane protein fractions. Detection of GAPDH expression was included as a control. (C) Cells were pre-treated with lovastatin and then incubated with 30 U/mL Epo. Cell proliferation was assessed using the MTT assay. (D) CaLo and InBl cells were cultured in FBS-free medium (w/o FBS), in FBS-free medium containing $15 \mathrm{U} / \mathrm{ml} \mathrm{SCF}$ (w/o FBS + SCF) or in medium supplemented with 10\% FBS as a control (FBS) for 14 days. Cell viability was evaluated by an MTT assay. (E) To demonstrate that the SCF-induced survival effect was mediated by c-Kit, cells were transfected with siRNA for transient inhibition of c-Kit gene expression (c-Kit) or with a non-related siRNA (scrambled) as a negative control. Expression of c-Kit was evaluated by western blotting and detection of GAPDH expression was included as a control. (F) Cells were transfected with specific c-Kit siRNA, non-related siRNA (scrambled) or left untreated. Following this, the cells were cultured in FBS-free medium containing $15 \mathrm{U} / \mathrm{ml} \mathrm{SCF}$ for 7 days. Cell viability was evaluated by an MTT assay. ${ }^{*} \mathrm{P}<0.05$, compared with untreated control values. For (A), (C) and (F), values represent the mean of three independent experiments and the error bars indicate the SEM. Epo, erythropoietin; SCF, stem cell factor; SEM, standard error of the mean; FBS, foetal bovine serum; siRNA, small interfering RNA. 
A

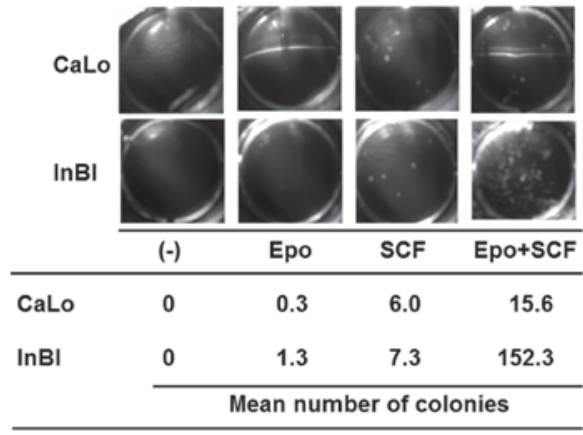

B

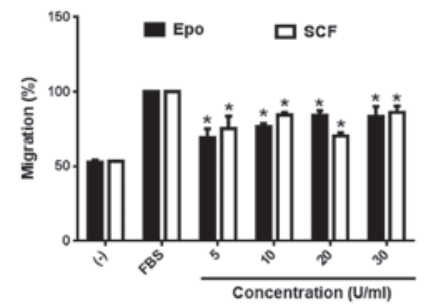

C

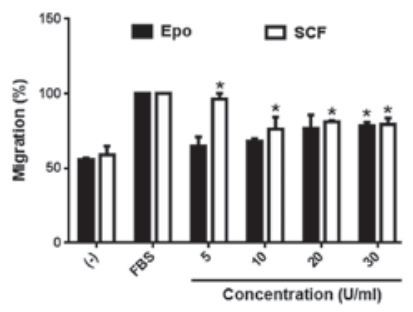

Figure 3. Effect of Epo and SCF on anchorage independent growth and cell migration. (A) Anchorage independent cell growth was evaluated by soft agar colony formation assays. Cells were seeded in soft agar containing Epo (30 U/ml), SCF $(15 \mathrm{U} / \mathrm{ml})$ or a combination of Epo and SCF (Epo+SCF) a the indicated concentrations, for 7 days. Colonies were stained and counted. A representative photograph of the colonies is shown. Data represent the mean of three independent experiments. Migration of (B) CaLo and (C) InBl cells was evaluated by Boyden chamber assays. The indicated concentrations of Epo and SCF were loaded into the lower chambers and the cells were allowed to migrate for $24 \mathrm{~h}$. As a negative control, FBS-free culture medium (-) was used. Medium supplemented with $10 \%$ FBS (FBS) was included as a positive control. Data represent the mean of three independent experiments and the error bars indicate the SEM. ${ }^{*} \mathrm{P}<0.05$, compared with negative controls. Epo, erythropoietin; SCF stem cell factor; SEM, standard error of the mean; FBS, foetal bovine serum.

vations suggest that SCF protects cells from starvation-induced death by activating its corresponding receptor, c-Kit.

Anchorage independent cell growth and migration are induced by a combination of Epo/SCF. Anchorage independent cell growth has been associated with metastatic potential. Therefore, it was next evaluated if Epo and/or SCF would be able to stimulate the formation of cell colonies in soft agar. The results are demonstrated in Fig. 3A. Administration of Epo or SCF alone produced a modest number of colonies in the cell lines. By contrast, the combination of Epo and SCF promoted a significant increase of cell colonies in CaLo and a highly significant increment of cell colony numbers in InBl cells. Cell migration is fundamental for tumour dissemination. Therefore, to investigate whether activation of EpoR and c-Kit induces cell migration, the cell lines were seeded on the upper insert of Boyden chambers, migration was tested in the presence of 5, 10, 20 and $30 \mathrm{U} / \mathrm{ml}$ of either Epo or SCF. As demonstrated in
Fig. 3B, CaLo cells were stimulated to migrate by Epo and SCF at the various different doses tested. The response of InBl cells was significantly higher at $5 \mathrm{U} / \mathrm{ml}$ of SCF, while only the higher concentration of Epo $(30 \mathrm{U} / \mathrm{ml})$ induced a significant number of migrating cells (Fig. 3C). Our results appear to indicate that Epo and SCF alone are associated with migration but not with clonal expansion of cervical tumours. This was in contrast with the combination of Epo/SCF, that was able to stimulate migration and colony formation.

Following this, we investigated the effect of a combination of Epo and SCF on the migratory behaviour of InBl cells. Our results demonstrate that the cell lines respond to Epo and SCF, thus for the following experiments, only $\mathrm{InBl}$ cells were tested. Three different combinations (10/20, 10/10 and 30/30 U/ml) of Epo/SCF were used. As observed in Fig. 4A, all of the combinations induced the migration of InBl, being 10/20 significantly higher. This particular combination was subsequently compared with the effect of the growth factors alone. The results are demonstrated in Fig. 4B. The proportion of cells migrating as a response to Epo alone was significantly lower than that induced by the combination of cytokine factors. By contrast, the proportion of migrating cells in the presence of SCF was comparable to that induced by the combination of growth factors. Our results appear to suggest that SCF is a strong inducer of migration. However, as described above, we identified that only $11.4 \%$ of InBl cells express c-Kit. Therefore, in order to corroborate that cells expressing c-Kit would in turn migrate as a response to $\mathrm{SCF}$, we isolated $\mathrm{c}-\mathrm{Kit}^{+}$cells by utilising paramagnetic microbeads conjugated to monoclonal anti-human CD117 antibody (Fig. 4C). The c-Kit ${ }^{+}$and $\mathrm{c}-\mathrm{Kit}^{-}$cells were tested in a migration assay in the presence of Epo, SCF or Epo/SCF (Fig. 4D). In all of the conditions the proportion of migrating $\mathrm{c}-\mathrm{Kit}^{+}$cells was significantly higher than the proportion of c-Kit cells. Additionally, migration induced by the combination of Epo/SCF was superior to that produced by SCF alone in the c-Kit ${ }^{+}$cells.

Migration is mediated by the activation of JAK2/STAT5 and ERK1/2. Our observations suggest that SCF, Epo and Epo/SCF induce the migration of cervical cancer cells. Binding of soluble SCF to c-Kit and Epo to its receptor activates three main signalling pathways. Thus, in an attempt to ascertain the participation of each cascade in migration, the cells were pre-incubated with Tyrphostin AG490, Wortmannin and U0126 ethanolate to inhibit JAK2, PI3K and ERK1/2, respectively and then tested in a migration assay. As depicted in Fig. 5A, migration induced by Epo, SCF and the combination of Epo/SCF was significantly reduced by inhibiting JAK2 phosphorylation. Conversely, inhibition of PI3K resulted in a modest decrement of migrating cells. By contrast, migration induced by Epo, SCF and Epo/SCF was abrogated by the inhibition of ERK1/ERK2. This observation strongly suggests that migration is regulated by JAK2-mediated signalling and the MAPK/ERK pathway. To corroborate the activation of these signalling pathways, we evaluated the Epo-, SCF- and Epo/SCF-mediated phosphorylation of STAT5 and ERK $1 / 2$ in a time-course experiment. As demonstrated in Fig. 5B, activation of STAT5 was evident only 1 min following stimulation with Epo, showing maximum phosphorylation at $30 \mathrm{~min}$. Stimulation with SCF induced a rapid activation of STAT5, presenting a maximum at 1 min following stimulation, with this response decaying after $30 \mathrm{~min}$. Activation of STAT5 
A

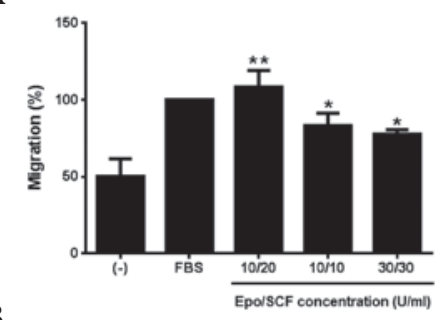

B

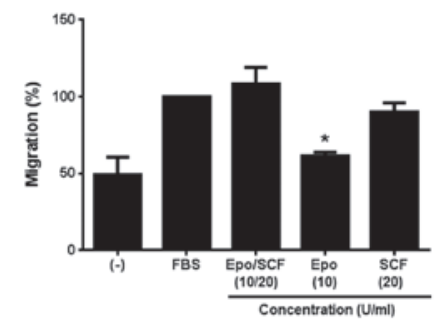

C

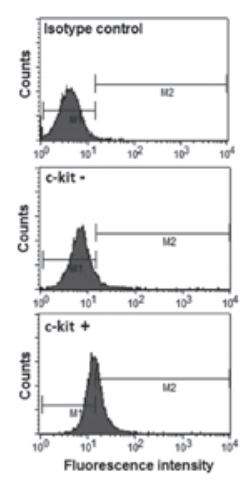

D

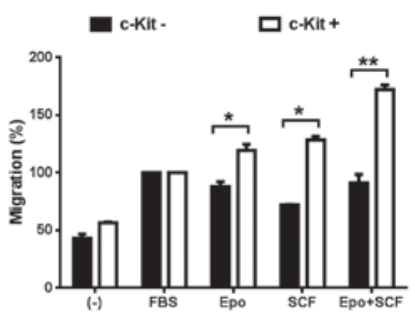

Figure 4. Induction of migration by co-stimulation with Epo and SCF. (A) InBl cells were co-stimulated with the indicated concentrations of Epo and SCF (Epo/SCF) in Boyden chambers for $24 \mathrm{~h}$. FBS-free medium (-) and medium supplemented with 10\% FBS were included as a negative and positive control, respectively. ${ }^{*} \mathrm{P}<0.05 ;{ }^{* *} \mathrm{P}<0.005$, compared with the negative controls. (B) InBl cells migration was evaluated in the presence of the combination of Epo/SCF that produced the highest migration effect $(10 / 20 \mathrm{U} / \mathrm{ml})$ and compared with the effect of either cytokine alone. As a negative control, FBS-free medium (-) was used. Medium supplemented with $10 \%$ FBS was included as a positive control. ${ }^{*} \mathrm{P}<0.05$, compared with cells stimulated with Epo/SCF. (C) c-Kit expressing InBl cells were isolated using anti-human CD117 (c-Kit)-conjugated paramagnetic microbeads. Isolated c-Kit expressing cells (c-Kit $\left.{ }^{+}\right)$, and unlabelled cells (c-Kit) were analysed by flow cytometry. As a negative control c-Kit expressing cells were incubated with a primary isotype control antibody instead of anti-c-Kit. (D) Isolated c-Kit expressing (c-Kit $\left.{ }^{+}\right)$and unlabelled (c-Kit) InBl cells were assessed in a migration assay in the presence of Epo (10 U/ml), SCF $(20 \mathrm{U} / \mathrm{ml})$ or a combination of Epo and SCF (Epo+SCF; 10/20 U/ml). As a negative control, FBS-free culture medium (-) was used. Medium supplemented with $10 \%$ FBS (FBS) was included as a positive control. ${ }^{*} \mathrm{P}<0.05$; ${ }^{* *} \mathrm{P}<0.005$, comparing c-Kit with c-Kit ${ }^{+}$cells. For (A), (B) and (D) data represent the mean of three independent experiments and the error bars indicate the SEM. Epo, erythropoietin; SCF, stem cell factor; FBS, foetal bovine serum; SEM, standard error of the mean.

A

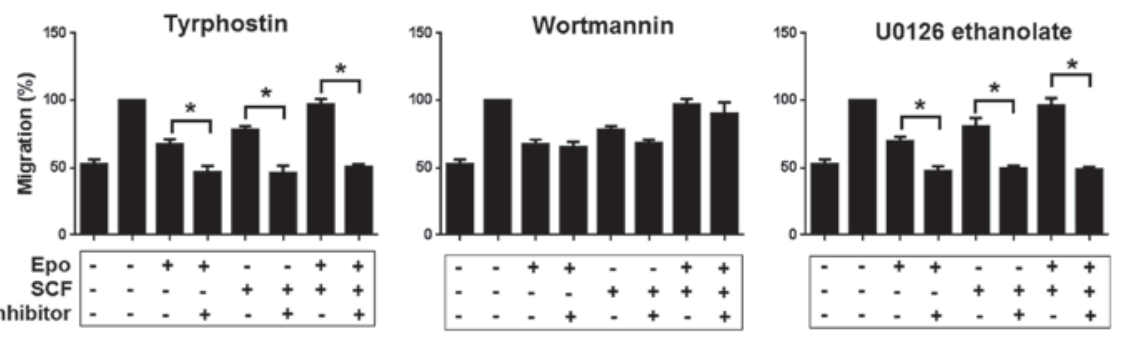

B

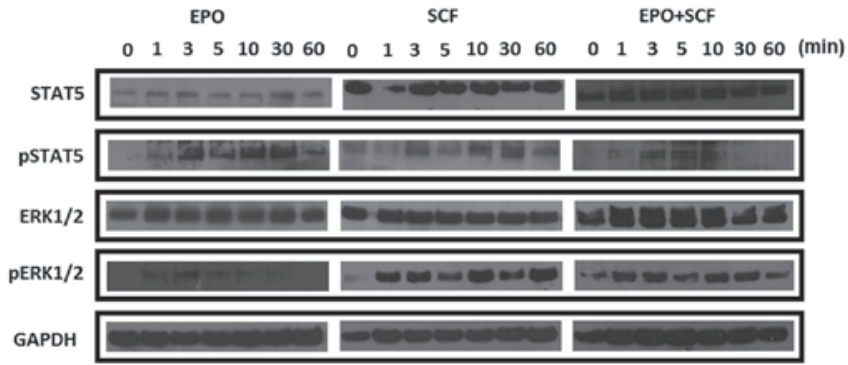

Figure 5. Effect of inhibition of different signalling pathways on Epo/SCF-induced migration. (A) To inhibit phosphorylation of JAK2, InB1 cells were pre-incubated with $10 \mu \mathrm{M}$ Tyrphostin AG490 diluted in ethanol for $24 \mathrm{~h}$. PI3K was inhibited by pre-incubating the cells with $100 \mathrm{nM}$ Wortmannin diluted in DMSO for $24 \mathrm{~h}$. To inhibit ERK1/2 kinases, the cells were pre-incubated with $20 \mu \mathrm{M}$ U0126 ethanolate diluted in DMSO for $24 \mathrm{~h}$. Migration induced by Epo $(10 \mathrm{U} / \mathrm{ml})$, SCF $(20 \mathrm{U} / \mathrm{ml})$ or a combination of Epo and SCF was then evaluated by Boyden chamber assays. All values reported represent the mean of three independent experiments and the error bars indicate the SEM. " $\mathrm{P}<0.05$ comparing cells pre-incubated with the indicated inhibitors with untreated cells. (B) Western blot analysis of STAT5 and ERK1/2 activation in response to Epo, SCF, and Epo/SCF stimulation. InBl cells were incubated with Epo (10 U/ml), SCF $(20 \mathrm{U} / \mathrm{ml})$ or Epo/SCF (10/20 U/ml). Protein lysates $(30 \mu \mathrm{g})$ were analysed by $10 \%$ SDS-PAGE. Specific proteins were detected using antibodies to STAT5, pSTAT5, ERK1/2 and pERK1/2. GAPDH detection was included as a control. A representative blot from triplicate experiments is presented. Epo, erythropoietin; SCF, stem cell factor; JAK2, Janus kinase 2; PI3K, phosphatidylinositol-3 kinase; ERK, extracellular signal-related kinase; SEM, standard error of the mean; STAT5, signal transducer and activator of transcription-5; pSTAT5, phospho-STAT5; pERK, 1/2 phospho-ERK1/2. 
was only detected at 3,5 and 10 min following treatment with the combination of Epo/SCF. Unlike STAT5, phosphorylation of ERK1/2 was weakly induced by Epo. By marked contrast, $\mathrm{SCF}$, as well as the combination of Epo/SCF, promoted a strong phosphorylation of ERK1/2 from 1 min following treatment. Notably, ERK1/2 activation persisted at high levels until the end of the time-course experiment $(60 \mathrm{~min})$.

\section{Discussion}

Experimental evidence demonstrating that Epo contributes to cellular proliferation of human cancer cells is expanding. Accordingly, Epo has been recently associated with proliferation of renal cell carcinoma (22), melanoma (23), head and neck cancer (24) and prostate carcinoma (25). In addition, we demonstrated that Epo promotes cell proliferation of three cervical cancer-derived cell lines (17). In the present study, it was observed that Epo stimulates proliferation of two more cervical cancer cell lines. These observations suggest that Epo-induced proliferation is a common feature of this type of cancer. On the other hand, expression of c-Kit receptor has been identified in cervical cancer tissue samples and cell lines (26). In this study, we have detected the expression of c-Kit in two cervical cancer-derived cell lines. However, it has been observed that only a small proportion $(<13 \%)$ of the cells were positive for the expression of the receptor at the cell surface. In spite of the low number of cells expressing c-Kit, SCF induced the survival of the cells when they were cultivated in the absence of FBS. Activation of the SCF/c-Kit axis is crucial for the survival of various types of cells, including hematopoietic cells (27), mast cells (28), embryonic stem cells (29) and ovarian cancer cells (30). This observation indicates that the activation of the c-Kit receptor in cervical cancer cells contributes to the survival of cells exposed to unfavourable conditions.

To further study the potential for SCF and Epo to support cervical cancer cells growth, an anchorage independent cell growth assay was set. The results demonstrated that individual administration of Epo or SCF induced a limited number of colonies. However, the combination of Epo/SCF produced a significantly higher number of colonies, particularly in InBl cells. Previous studies have demonstrated that SCF is able to increase the colony-forming potential of colon carcinoma cells (31), and that Epo augments the number of colonies in a modified, c-Kitexpressing breast cancer cell line (32). However, the coordinated effect of Epo and SCF on the colony-forming potential of tumour cells had not been described until now. Anchorage independent growth has been associated with metastatic potential, but cell migration is considered to be the first step in metastasis. Of note, in this study it was identified that migration of cervical cancer cells was stimulated by Epo and SCF alone, but was significantly enhanced by their co-administration. These results are consistent with an earlier study, which demonstrated that Epo induced the migration of HeLa cells, acting as a chemoattractant under serum-starved conditions (20). Similarly, induction of cell migration by SCF has been reported in colon carcinoma cells (31). The combined effect of Epo and SCF on the migration of cancer cells had not been investigated until now.

The results in the present study strongly suggest the cytokines Epo and SCF have a cooperative effect in cervical cancer. The coalition of Epo and SCF was initially observed during the generation of erythroid blast and colony forming units, where it was revealed that c-Kit, via the interaction with the extended box 2 region of EpoR, triggered the induction of phosphorylation of EpoR's tyrosine residue (33). Additionally, the synergistic interactions between Epo and SCF appears to be due to co-signalling. EpoR and c-Kit share three basic signalling pathways, JAK/STAT, PI3K/AKT and MAPK/ERK. In the present study, it was identified that the inhibition of JAK2 eliminated Epo/SCF-induced migration. In this context, Hong et al (12) reported that the JAK2 binding site in the EpoR is essential for co-signalling with c-Kit receptor. Furthermore, c-Kit has been demonstrated to cross-talk with the JAK2/STAT5 axis to promote haematopoiesis (34). In this study we observed the activation of STAT5, however, there was no difference between the level of STAT5 phosphorylation induced by either cytokine alone or in combination. These observations indicate that the JAK2/STAT5 system, although not directly activated by these cytokines, is crucial in promoting migration as a response to Epo, SCF and Epo/SCF stimulation in cervical cancer cells.

Similarly, obstruction of the MAPK/ERK pathway using the ERK1/2-specific inhibitor U0126 ethanolate, eliminated Epo-, SCF- and Epo/SCF-induced cell migration. Consistent with these data, an earlier study identified that the migration of HeLa cells was induced by Epo in a MAPK-dependent manner (20). The authors also described that the activation of this pathway was, in turn, dependent on JAK2 activity. In the present study, it was observed that co-administration of Epo and SCF significantly increased migration of InBl cultures enriched with c-Kit ${ }^{+}$cells. SCF and Epo have been demonstrated to induce the continuous activation of ERK1/2 in erythroid cells synergistically (35). In this study, it was revealed that co-stimulation with SCF and Epo produced a sustained activation of ERK1/2 in InBl cells. Notably, treatment with Epo caused a modest and transient activation of ERK1/2, whereas treatment with SCF prompted a sustained activation of these kinases. These results indicate that Epo/SCF co-stimulation of InBl cells migration is regulated by the JAK2/STAT5 axis in coordination with a sustained activation of ERK1/2.

In summary, we have demonstrated that co-stimulation of cervical cancer cells with Epo and SCF promotes migration and anchorage independent cell growth, which are effects that are superior to that promoted by either cytokine alone. In addition, our results suggest that co-signalling from EpoR and c-Kit converge on JAK2/STAT5 activation, being that this signalling pathway is an important regulator of migration. Of note, stimulation with SCF alone as well as Epo/SCF in combination, induced a sustained activation of ERK1/2 and inhibition of ERK1/2 resulted in the abrogation of migration. Metastasis is a complex issue and these results provide important insights into how co-signalling from different receptors induces migration, and suggests that migration may be regulated by a variety of signalling pathways. Therefore, future studies investigating multiple regulatory cascades participating in migration would facilitate the development of more efficacious therapeutic approaches in the treatment of cancer.

\section{Acknowledgements}

This study was supported by grants from CONACyT (grant: 152492) and PAPIIT (grant: IN209613). The present work 
was performed in partial fulfilment of the requirements for the $\mathrm{PhD}$ degree in Ciencias Biologicas of Cristina Aguilar at the Universidad Nacional Autonoma de México, with doctoral scholarships from CONACyT (Reg: 49102), ICyTDF (Reg: BI12-179), and COMECyT (Reg: 12BCD0052-I). The authors thank the Posgrado en Ciencias Biologicas, UNAM for academical support.

\section{References}

1. Witthuhn BA, Quelle FW, Silvennoinen O, Yi T, Tang B, Miura $\mathrm{O}$ and Ihle JN: JAK2 associates with the erythropoietin receptor and is tyrosine phosphorylated and activated following stimulation with erythropoietin. Cell 74: 227-236, 1993.

2. Bao H, Jacobs-Helber SM, Lawson AE, Penta K, Wickrema A and Sawyer ST: Protein kinase B (c-Akt), phosphatidylinositol 3-kinase, and STAT5 are activated by erythropoietin (EPO) in HCD57 erythroid cells but are constitutively active in an EPO-independent, apoptosis-resistant subclone (HCD57-SREI cells). Blood 93: 3757-3773, 1999.

3. Sokolovsky M, Nam H, Fleming MD, Haase VH, Brugnara C and Lodish HF: Ineffective erythropoiesis in Stat $5 \mathrm{a}(-/-) 5 \mathrm{~b}(-/-)$ mice due to decreased survival of early erythroblasts. Blood 98 : 3261-3273, 2001

4. Damen JE, Wakao H, Miyajima A, Krosl J, Humphries RK, Cutler RL and Krystal G: Tyrosine 343 in the erythropoietin receptor positively regulates erythropoietin-induced cell proliferation and Stat5 activation. EMBO J 14: 5557-5568, 1995.

5. Blume-Jensen P, Janknecht R and Hunter T: The kit receptor promotes cell survival via activation of PI 3-kinase and subsequent Akt-mediated phosphorylation of Bad on Ser136. Curr Biol 8: 779-782, 1998.

6. Krystal GW, DeBerry CS, Linnekin D and Litz J: Lck associates with and is activated by Kit in a small cell lung cancer cell line: inhibition of SCF-mediated growth by the Src family inhibitor PP1. Cancer Res 58: 4660-4666, 1998.

7. Sundström M, Alfredsson J, Olsson N and Nilsson G: Stem cell factor-induced migration of mast cells requires p38 mitogenactivated protein kinase activity. Exp Cell Res 267: 144-151, 2001

8. Maddens S, Charruyer A, Plo I, Dubreuil P, Berger S, Salles B, Laurent $G$ and Jaffrézou JP: Kit signalling inhibits the sphingomyelin-ceramide pathway through PLC gamma 1: implication in stem cell factor radioprotective effect. Blood 100: 1294-1301, 2002.

9. Trieselmann NZ, Soboloff J and Berger SA: Mast cells stimulated by membrane-bound, but not soluble, steel factor are dependent on phospholipase C activation. Cell Mol Life Sci 60: 759-766, 2003.

10. Wu H, Klingmüller U, Besmer $\mathrm{P}$ and Lodish HF: Interaction of the erythropoietin and stem-cell-factor receptors. Nature 377: 242-246, 1995.

11. Wu H, Klingmüller U, Acurio A, Hsiao JG and Lodish HF: Functional interaction of erythropoietin and stem cell factor receptors is essential for erythroid colony formation. Proc Natl Acad Sci USA 94: 1806-1810, 1997.

12. Hong L, Ramdas B, Chen J, Harris C, Wojchowski DM and Kapur R: KIT associated intracellular tyrosines play an essential role in EpoR co-signalling. Cell Signal 20: 1513-1520, 2008.

13. Farrell $F$ and Lee A: The erythropoietin receptor and its expression in tumor cells and other tissues. Oncologist 9 (Suppl 5): 18-30, 2004.

14. Horie K, Fujita J, Takakura K, Kanzaki H, Suginami H, Iwai M, Nakayama $\mathrm{H}$ and Mori T: The expression of c-kit protein in human adult and fetal tissues. Hum Reprod 8: 1955-1962, 1993.

15. Shenouda G, Mehio A, Souhami L, Duclos M, Portelance L, Belenkov A and Chow T: Erythropoietin receptor expression in biopsy specimens from patients with uterine cervix squamous cell carcinoma. Int J Gynecol Cancer 16: 752-756, 2006.

16. Caceres-Cortes JR, Alvarado-Moreno JA, Waga K, Rangel-Corona R, Monroy-Garcia A, Rocha-Zavaleta L, Urdiales-Ramos J, Weiss-Steider B, Haman A, Hugo P, et al: Implication of tyrosine kinase receptor and steel factor in cell density-dependent growth in cervical cancers and leukemias. Cancer Res 61: 6281-6289, 2001.

17. Lopez TV, Lappin TR, Maxwell P, Shi Z, Lopez-Marure R, Aguilar C and Rocha-Zavaleta L: Autocrine/paracrine erythropoietin signalling promotes JAK/STAT-dependent proliferation of human cervical cancer cells. Int J Cancer 129: 2566-2576, 2011.
18. Mohyeldin A, Lu H, Dalgard C, Lai SY, Cohen N, Acs G and Verma A: Erythropoietin signaling promotes invasiveness of human head and neck squamous cell carcinoma. Neoplasia 7: 537-543, 2005

19. Lester RD, Jo M, Campana WM and Gonias SL: Erythropoietin promotes MCF-7 breast cancer cell migration by an ERK/mitogen-activated protein kinase-dependent pathway and is primarily responsible for the increase in migration observed in hypoxia. J Biol Chem 280: 39273-39277, 2005.

20. Hamadmad SN and Hohl RJ: Erythropoietin stimulates cancer cell migration and activates RhoA protein through a mitogen-activated protein kinase/extracellular signal-regulated kinase-dependent mechanism. J Pharmacol Exp Ther 324: $1227-1233,2008$

21. Fraser JK, Lin FK and Berridge MV: Expression and modulation of specific, high affinity binding sites for erythropoietin on the human erythroleukemic cell line K562. Blood 71: 104-109, 1988.

22. Fujisue $Y$, Nakagawa $T$, Takahara $K$, Inamoto T, Kiyama S, Azuma $\mathrm{H}$ and Asahi M: Induction of erythropoietin increases the cell proliferation rate in a hypoxia-inducible factor-1-dependent and -independent manner in renal cell carcinoma cell lines. Oncol Lett 5: 1765-1770, 2013.

23. Kumar SM, Zhang G, Bastian BC, Arcasoy MO, Karande P, Pushparajan A, Acs G and Xu X: Erythropoietin receptor contributes to melanoma cell survival in vivo. Oncogene 31: 1649-1660, 2012.

24. Steinmann K, Richter AM and Dammann RH: Epigenetic silencing of erythropoietin in human cancers. Genes Cancer 2: 65-73, 2011.

25. Jeong JY, Hoxhaj G, Socha AL, Sytkowski AJ and Feldman L: An erythropoietin autocrine/paracrine axis modulates the growth and survival of human prostate cancer cells. Mol Cancer Res 7: 1150-1157, 2009.

26. Inoue M, Kyo S, Fujita M, Enomoto $T$ and Kondoh G: Coexpression of the c-kit receptor and the stem cell factor in gynecological tumors. Cancer Res 54: 3049-3053, 1994.

27. Kapur R, Cooper R, Zhang L and Williams DA: Cross-talk between alpha(4)beta(1)/alpha(5)beta(1) and c-Kit results in opposing effect on growth and survival of hematopoietic cells via the activation of focal adhesion kinase, mitogen-activated protein kinase, and Akt signalling pathways. Blood 97: 1975-1981, 2001.

28. Yang FC, Kapur R, King AJ, Tao W, Kim C, Borneo J, Breese R, Marshall M, Dinauer MC and Williams DA: Rac2 stimulates Akt activation affecting $\mathrm{BAD} / \mathrm{Bcl}-\mathrm{XL}$ expression while mediating survival and actin function in primary mast cells. Immunity 12 : 557-568, 2000.

29. Fraser L, Taylor AH and Forrester LM: SCF/KIT inhibition has a cumulative but reversible effect on the self-renewal of embryonic stem cells and on the survival of differentiating cells. Cell Reprogram 15: 259-268, 2013.

30. Liu L, Zhang X, Do C, Zhang X, Hou N, Zhao D, Sun J, Li L, Wang $X$ and Ma C: MEK1-independent activation of MAPK and MEK1-dependent activation of p70 S6 kinase by stem cell factor (SCF) in ovarian cancer cells. Biochem Biophys Res Commun 382: 385-389, 2009

31. Bellone G, Carbone A, Sibona N, Bosco O, Tibaudi D, Smirne C, Martone T, Gramigni C, Camandona M, Emanuelli G and Rodeck U: Aberrant activation of c-kit protects colon carcinoma cells against apoptosis and enhances their invasive potential. Cancer Res 61: 2200-2206, 2001

32. Shi Z, Hodges VM, Dunlop EA, Percy MJ, Maxwell AP, El-Tanani $\mathrm{M}$ and Lappin TR: Erythropoietin-induced activation of the JAK2/STAT5,PI3K/Akt, and Ras/ERK pathways promotes malignant cell behavior in a modified breast cancer cell line. Mol Cancer Res 8: 615-626, 2010.

33. Wu H, Klingmüller U, Bersmer P and Lodish HF: Interaction of the erythropoietin and stem-cell-factor receptors. Nature 377: 242-246, 1995.

34. Grebier F, Kerenyi MA, Kovacic B, Kolbe T, Becker V, Dolznig H, Pfeffer K, Klingmüller U, Müller M, Beug H, et al: Stat5 activation enables erythropoiesis in the absence of EpoR and Jak2. Blood 111: 4511-4522, 2008

35. Sui X, Krantz SB, You M and Zhao Z: Synergistic activation of MAP kinase (ERK1/2) by erythropoietin and stem cell factor is essential for expanded erythropoiesis. Blood 92: 1142-1149, 1998. 